\section{Expected and unexpected enteric viruses in the UK dog population; a comprehensive survey by qPCR}

\section{Sarah Caddy ${ }^{2,1}$, Aoife McElroy', Ian Goodfellow ${ }^{1}$}

University of Cambridge, Cambridge, UK

Imperial College London, London, UK

A number of novel viruses have been associated with canine gastroenteritis in recent years, including astroviruses, noroviruses, vesiviruses and kobuviruses. Viral gastroenteritis in dogs worldwide is acknowledged as being predominantly caused by canine parvovirus (CPV) and canine enteric coronavirus (CECOV), with the contribution of recently identified viruses associated with gastroenteritis uncertain. Norovirus and astrovirus strains that infect man cause significant levels of disease in humans and yet canine strains of these viruses have not previously been identified in any dogs in the UK. This study sought to investigate the prevalence and potential significance of both commonly identified viruses, and novel enteric viruses in the UK dog population.

Sixty-nine dogs with severe gastroenteritis were enrolled in the study with an additional 180 dogs recruited as controls.
With owner consent, stool samples were collected and nucleic acid was extracted. Quantitative PCR targeting a range of canine viruses associated with gastroenteritis was used to screen all stool samples. Confirmation of all positive samples was achieved by sequencing.

CPV was identified most frequently in the study population as expected; $14.5 \%$ dogs with gastroenteritis were positive. Sequencing showed $30 \%$ of CPV strains were type-2a and $70 \%$ were type-2b. These results confirm that type-2c is still not identifiable in the UK. CECoV was also detected in two cases with gastroenteritis; a similar prevalence level to previous reported. As for viruses for which commercial tests are not available, a single dog with gastroenteritis dog was positive for kobuvirus, and surprisingly, canine astrovirus (CaAstV) was detected in four dogs with gastroenteritis. Two positive CaAstV cases were puppies under 10 weeks old, also coinfected with CPV. The other two cases were in adult dogs with no other viral aetiology identified. Molecular characterisation showed only $80 \%$ amino acid identity between CaAstV strains, identifying significant genetic variation.

In summary, this study presents an overview of the prevalence and diversity of canine enteric viruses in the UK in 20122014. Importantly, this suggests that CaAstV may be a significant and hitherto unrecognized cause of canine gastroenteritis, in addition to the numerous other viruses identified. It is recommended that veterinarians are aware of these emerging viruses for which there are no commercial tests; isolation of suspicious cases is always advised.

\section{Can we diagnose extra-hepatic biliary obstruction (EHBO) in cats with cholangitis without exploratory laparotomy?}

\section{Jack Lawson', Jesabelle Kibanda', Barbara Glanemann', Oliver Garden', Severine Tasker ${ }^{3}$, Michael Day ${ }^{3}$, Penny Watson ${ }^{2}$}

Royal Veterinary College, London, UK Cambridge University, Cambridge, UK University of Bristol, Bristol, UK

Cholangitis is one of the most common histological diagnoses in feline liver disease and may occur with or without concurrent $\mathrm{EHBO}$. The presence of EHBO informs treatment, but identifying $\mathrm{EHBO}$ without exploratory laparotomy is challenging. Ultrasonography commonly shows biliary dilation, but is not diagnostic in some cases, and the assumption that ultrasound is $100 \%$ sensitive for EHBO may lead to cases being misdiagnosed. The aim of this study was to identify distinctive changes on histology, serum biochemistry and haematology which may indicate the presence of obstructive disease in addition to ultrasonography.

The records of three veterinary hospitals were searched from 2002-2012 for cats with a histopathological diagnosis of cholangitis/cholangiohepatitis. Cats were included in the cholangitis associated with EHBO group (C+EHBO group) if biliary obstruction was confirmed at laparotomy/necropsy. Cats with surgical confirmation of biliary patency or where there was no suspicion of $\mathrm{EHBO}$ on ultrasonography were included in the cholangitis group ( $C$ group). Serum biochemistry, haematology, coagulation profile and imaging findings were recorded, and histopathology slides were reviewed by a pathologist. Data from the two groups were compared using Fisher's exact test or the Mann-Whitney $U$ test.

Forty cats (C group) and seventeen cats ( $\mathrm{C}+\mathrm{EHBO}$ group) were identified. 9/40 cats in $C$ group had surgical confirmation of biliary patency (CS). Median ALKP, AST, GGT and total bilirubin (TBil) were elevated in both groups but only TBil differed significantly (C+EHBO group: $87.1 \mu \mathrm{mol} / \mathrm{L}, \mathrm{C}$ group: $19.7 \mu \mathrm{mol} / \mathrm{L}, \mathrm{p}=0.016)$. There was no difference in haematology or coagulation profile between groups. Fewer cats in the $\mathrm{C}+\mathrm{EHBO}$ group had sonographical abnormalities of the liver parenchyma (C+EHBO group: 25\% [4/16], C group: $72 \%$ [29/40 including 8/9 CS], $p=0.002$ ) but cats in this group demonstrated more biliary abnormalities, particularly dilation $(>5 \mathrm{~mm})$ of the common bile duct (CBD) (C+EHBO group: $94 \%$ [15/16], C group 22.5\% [9/40 including 4/9 CS], $p=<0.001)$. Histopathological features in both groups were similar, but there was increased prevalence of neutrophilic bile ductule infiltration in the $\mathrm{C}+\mathrm{EHBO}$ group ( $\mathrm{C}+\mathrm{EHBO}$ group: $35 \%$ [6/16], C group: 5\% [2/40 including 1/9 CS], $p=0.029$ ).

In conclusion, differentiating cats with EHBO remains 